\title{
Editorial: ASE 2013 Conference Trip Report
}

\author{
Robert J. Hall
}

Received: 27 November 2013 / Accepted: 9 December 2013 / Published online: 24 January 2014 (C) Springer Science+Business Media New York 2014

This issue puts together two regular manuscripts (the first two) with two manuscripts that complete last year's special multi-issue on Innovative Tools, guest edited by John Grundy and John Hosking. In addition to those research contributions, which are the true content of this issue, of course, I include below my usual report on attending the ASE (2013) Conference.

- In "Exploring optimization and caching for efficient collection operations", by Nerella, Surapaneni, Madria, and Weigert, the authors provide a novel technique for query optimization and caching in support of first class operations on collection types. They describe an implementation within the Java Query Language framework using AspectJ that performs better than a JQL benchmark from the literature.

- It is becoming common to include machine learning components in software products for purposes of tailoring, prediction, or optimization. And yet it is difficult to select appropriate learning algorithms for a particular task or product taking into account non-functional as well as functional requirements for the product. Lavesson, Boeva, Tsiporkova, and Davidsson describe "A method for evaluation of learning components" as well as two significant case studies of applying it.

- (SI on Innovative Tools Paper) Nguyen, Robbins, Banerjee, and Memon describe a novel framework for testing the user interface of an application in "GUITAR: an innovative tool for automated testing of GUI-Driven software". Its innovative plug-in based architecture supports flexibility and extensibility, allowing it to fit into larger tool chains.

- (SI on Innovative Tools Paper) Finally, in "Behind the scenes in SANTE: a combination of static and dynamic analyses", Chebaro, Cuoq, Kosmatov, Marre, Pacalet, Williams, and Yakobowski describe a tool architecture that allows one to combine

R. J. Hall (凶)

AT\&T Labs Research, Bedminster, NJ 07921, USA

e-mail: Bob.ASEJ@gmail.com 
several, quite distinct $\mathrm{C}$ program verification tools together. It also analyzes their particular aspects and shows how their benefits combine.

\section{The 28th International Conference on Automated Software Engineering}

ASE 2013 was held November 11-15 in Palo Alto, CA, USA, at the Crowne Plaza Cabana Hotel. General Chair Ewen Denney of NASA Ames and his organizing committee provided a very rich conference in a nice venue with great weather.

The conference featured a couple of invited contributions as well as a panel discussion. Jeremy Frank of NASA Ames discussed the challenges of V\&V applied to automated planning systems, the prime example of such being those used in some of NASA's deep space missions. Given the domain complexity and high cost of errors, this is an interesting set of challenges to $V \& V$ techniques indeed. Joseph Hellerstein spoke on his group's work on the Bloom language and associated tools for cloud (distributed) computing. The panel discussion on "Big problems in industry" focused on various ways real world scale causes complexities and how various groups attack them.

This year's conference proceedings, selected from over 300 submissions, had a bumper crop of research full papers. In addition, it had an eight-paper "Experience Track", divided into software analysis papers and testing and verification papers. This relatively large set of experience papers compared to past years evinces both the health and relevance of ASE. The conference also had a large group of "New Ideas" papers, which have been termed "short papers" in past years, and a healthy and interesting "Tool Demonstrations" track. The demonstrations are a signature feature of ASE conferences, and this year's lively and interactive session did not disappoint. Rounding out the program, the "Doctoral Symposium" included five contributions, as well.

The conference banquet took place at the Computer History Museum in Mountain View, CA, USA. This was a real treat, especially for those of us old enough to have programmed on punch cards and personal computers from the 1970s. (Er, ... so I'm told.) It was a real opportunity to stroll through the history of computing technology and reminisce as well as learn. The food was good, too.

In this year's annual meeting of the ASE Steering Committee, David Lo of Singapore Management University was selected as General Chair of ASE 2016. The conference will be held in Singapore in September 2016. ASE 2014 will be in Västerås, Sweden in September (Ivica Crnkovic, General Chair), while ASE 2015 will be in Lincoln, Nebraska, USA (Myra Cohen, General Chair).

You are invited, as always, to email me atBob.ASEJ@gmail.com with your thoughts on this issue or the Journal in general. 